\title{
PROPAGATION OF TRANSIENT ELECTROMAGNETIC WAVES IN A LOSSY MAGNETOPLASMA HALF-SPACE WITH ARBITRARILY-ORIENTED MAGNETIC FIELD
}

\author{
M. Omid and M. Hayakawa \\ Department of Electronic Engineering, The University of Electro-Communications \\ Chofu Tokyo 182
}

\begin{abstract}
The time-dependent reflected and transmitted waveforms for an impulsive plane wave incident normally onto a lossy magnetoplasma half-space are computed by the use of a numerical inversion method of the Laplace transform. The temporal evolution of wave packets are analyzed for a two-component collisional (cold) plasma with the Earth's magnetic field, $\mathbf{B}_{0}$, being allowed to have an arbitrary direction. Curves are presented which show the dependence of the reflected and transmitted electric field components on the orientation of the Earth's magnetic field. Properties of the transmitted waveforms are discussed in three frequency bands; (i) quasi-free space modes, (ii) whistler mode and (iii) hydromagnetic wave modes. It is found that the arrival of signal delay for the whistler wave is inversely proportional to the cosine of the angle that $\mathbf{B}_{0}$ makes with the normal to the interface and the amplitudes of the electric field components are reduced in intensity for larger inclination of $\mathbf{B}_{0}$. Other features for the cases (ii) and (iii) are also discussed. The overall behavior of the wave packets suggests that the result may have some applications in diagnostic studies of magnetoplasma.
\end{abstract}

\section{Introduction}

The transient propagation of pulses in a homogeneous loss-free plasma half-space was formulated by Ishimaru (1967) many years ago, but no numerical results were presented. The impulse responses from a lossy anisotropic plasma half-space of a linearly polarized plane wave at normal incidence have later been evaluated numerically by Stanic and Skoric (1972). Hosono et al. (1989) have derived expressions for the transmitted wave field components with the Earth's magnetic field, $\mathbf{B}_{0}$, being allowed to have an arbitrary direction, but they were concerned with very high frequency waves only and neglected the effects of ions and collisions in their analysis. Recently, Omid et al. (1993) have reconsidered the problem and presented curves for the time-dependent transmitted waveforms in the entire frequency band with a more sophisticated model of anisotropic region, reperensntative of an actual $F_{2}$ plasma layer. The transient excitation by a delta function current source in a $F_{2}$ layer magnetoplasma have also been studied(Omid and Hayakawa, 1992, 1994) using a numerical inversion method of the Laplace transform (ILT). The same technique will be used here.

This paper is concerned with the problem of transmission / reflection of transient signals through / from a sharply bounded anisotropic medium. The medium is regarded as a twocomponent collisional (cold) plasma which can adequetely represent the ionospheric $\mathrm{F}_{2}$ layer with the Earth's magnetic field allowed to have an arbitrary direction. In Section 2, 
the time-harmonic solutions are obtained for an assumed linearly polarized wave having only the $x$-component as its electric field incident normally upon a plasma boundary. After the solution has been put into the desired form, suitable for numerical analysis, the impulse responses are evaluated via ILT, in Section 3. The numerical results are discussed for three different frequency regimes, namely, (i) quasi-free space modes, (ii) whistler mode and (iii) hydromagnetic wave modes. Finally, a brief summary of the main results obtained and conclusions are given in Section 4.

\section{Formulation}

\section{(a) Basic equations}

Transmission and reflection of a plane wave from a homogenous magnetoplasma bounded by a plane interface are considered. The superimposed magnetic field has an arbitrary orientation. Choosing a Cartesian coordinate system $(x, y, z)$, the interface between the free-space $(z<0)$ and ionosphere $(z>0)$ lies at the plane $z=0$ as indicated in Fig.1. Without loss of generality the earth's magnetic field $\mathbf{B}_{0}$ is assumed to be in the $x z$-plane, making an angle $\vartheta$ with $z$, i.e. $\mathbf{B}_{0}=B_{0}(\hat{\mathbf{x}} \sin \vartheta+\hat{\mathbf{z}} \cos \vartheta)$, where $\hat{\mathbf{x}}$ and $\hat{\mathbf{z}}$ are unit vectors in the positive $x$ and $z$ directions, respectively. In the following, the indices $i, r$, and $t$ denote the incident, reflected and transmitted waves, respectively. Suppose that a linearly polarized wave is normally incident upon a plasma boundary. As a result, a reflected wave is set up in the free-space and a transmitted wave in the plasma. Within the plasma two waves are generally generated, because of the double valued nature of the refractive index. Hence, the polarization of reflected and transmitted fields may have components in both $x$ and $y$ directions.

To obtain the electromagnetic fields, we regard the plasma as a medium which is homogeneous in both space and time and its electric permittivity is in the form of a tensor $\hat{\varepsilon}$ while the magnetic permeability is a scalar, then the two Maxwell's equations can be written as,

$$
\nabla \times \mathbf{E}=-\mu_{0} \frac{\partial \mathbf{H}}{\partial t}, \quad \nabla \times \mathbf{H}=\varepsilon_{0} \hat{\varepsilon} \frac{\partial \mathbf{E}}{\partial t}
$$

where $\mu_{0}$ and $\varepsilon_{0}$ are, respectively, the permeability and the permittivity of free-space. The components of the permittivity tensor $\hat{\varepsilon}$ in the rectangular frame of coordinate described above (see Fig.1) are given by a $3 \times 3$ matrix whose elements can be written as $\varepsilon_{i j}$ with $i, j=x, y, z$ to indicate its components (Budden, 1985). The explicit expressions for the elements of $\hat{\varepsilon}$ in a collisional two-component plasma consisting of electrons and one ion species can be found in Omid and Hayakawa (1994). Eliminating $\mathbf{H}$ from the Maxwell's equations (1), then the wave equation for the electric field vector can be expressed as,

$$
\nabla \times \nabla \times \mathbf{E}+\mu_{0} \varepsilon_{0} \hat{\varepsilon} \frac{\partial^{2} \mathbf{E}}{\partial t^{2}}=0 .
$$

Let us now seek for a plane wave solution of the form $\mathbf{E}(\omega, \mathbf{r})=\mathbf{E}_{0} \exp [j(\omega t-\mathbf{k} \cdot \mathbf{r})]$, where $\mathbf{E}_{0}$ is a constant vector and $\mathbf{k}$ is the propagation vector to be determined. Since the geometry and excitation are independent of $x$ and $y$ coordinates, the derivatives $\partial / \partial x, \partial / \partial y$ are zero and $\partial / \partial z=-j k_{z}$ for all field components. Hence, the vector wave equation in 


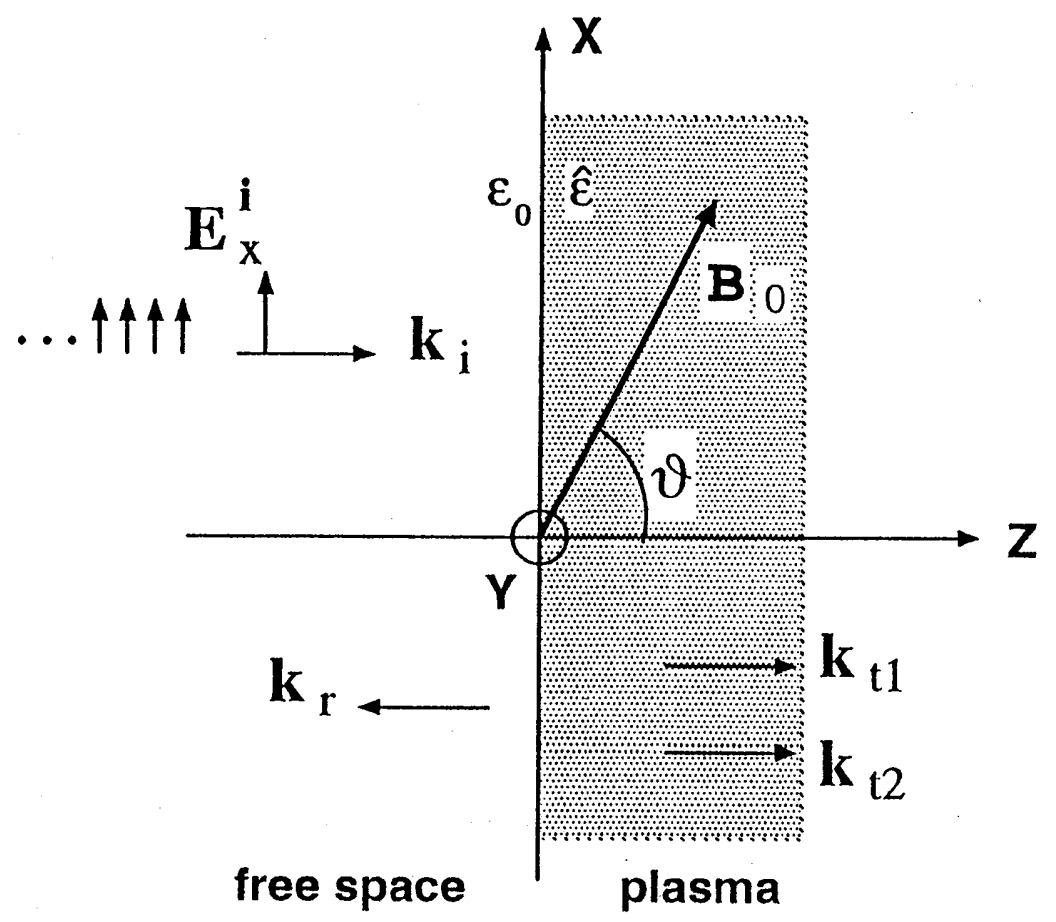

Fig. 1 The geometry of the problem. The Earth's magnetic field $B_{0}$ lying in $x-z$ plane, makes an angle $\vartheta$ with the $z$-axis (propagation direction $\mathrm{k}=k \hat{\mathbf{z}}$ ).

Table 1 Ionospheric parameters

\begin{tabular}{llll}
\hline parameter & value & parameter & normalized to $\omega_{p e}$ \\
\hline$f_{p e}$ & $10.0 \mathrm{MHz}$ & $\omega_{p e}$ & $2 \pi \times 10^{7} \mathrm{sec}^{-1}$ \\
$f_{p i}$ & $58.3 \mathrm{kHz}$ & $\omega_{p i}$ & $5.83 \times 10^{-3} \omega_{p e}$ \\
$f_{c e}$ & $1.0 \mathrm{MHz}$ & $\omega_{c e}$ & $0.10 \omega_{p e}$ \\
$f_{c i}$ & $34 \mathrm{~Hz}$ & $\omega_{c i}$ & $3.4 \times 10^{-6} \omega_{p e}$ \\
$f_{R}$ & $10.55 \mathrm{MHz} z$ & $\omega_{R}$ & $1.055 \omega_{p e}$ \\
$f_{L}$ & $9.55 \mathrm{MHz}$ & $\omega_{L}$ & $.955 \omega_{p e}$ \\
$f_{U H R}$ & $10.05 \mathrm{MHz} z$ & $\omega_{U H R}$ & $1.005 \omega_{p e}$ \\
$f_{L H R}$ & $5.80 \mathrm{kHz} z$ & $\omega_{L H R}$ & $5.80 \times 10^{-4} \omega_{p e}$ \\
$\nu_{e}$ & $1.0 \mathrm{kHz}$ & $\nu_{e}$ & $1.59 \times 10^{-5} \omega_{p e}$ \\
$\nu_{i}$ & $0.034 \mathrm{~Hz}$ & $\nu_{i}$ & $5.41 \times 10^{-10} \omega_{p e}$ \\
\end{tabular}

Ionospheric parameters for a day-time $F_{2}$ layer plasma, dominated by $O^{+}$, at altitude $z \simeq 300$ to $400 \mathrm{~km}$, with the electron number density $n_{e}=1.24 \times 10^{6} \mathrm{~cm}^{-3}$. In this table $f_{p e}$ and $f_{c e}$, the electron plasma and electron cyclotron frequencies; $f_{p i}$ and $f_{c i}$, the ion plasma and ion cyclotron frequencies; $f_{R}$ and $f_{L}$, the cutoff frequencies for right- and left- hand polarized waves; $f_{U H R}$ and $f_{L H R}$, the upper- and lower-hybrid resonance frequencies; and $\nu_{e}$ and $\nu_{i}$, the collision frequencies of the electrons and ions with the neutral particles (molecules). 
Eq.(2) for the components of the electric fields can be written in a matrix form in its simplified form as,

$$
-k_{z}^{2}\left[\begin{array}{c}
E_{x} \\
E_{y} \\
0
\end{array}\right]+\left(\frac{\omega}{c}\right)^{2}\left[\begin{array}{lll}
\varepsilon_{x x} & \varepsilon_{x y} & \varepsilon_{x z} \\
\varepsilon_{y x} & \varepsilon_{y y} & \varepsilon_{y z} \\
\varepsilon_{z x} & \varepsilon_{z y} & \varepsilon_{z z}
\end{array}\right]\left[\begin{array}{l}
E_{x} \\
E_{y} \\
E_{z}
\end{array}\right]=0
$$

where $c=\left(\mu_{0} \varepsilon_{0}\right)^{-1 / 2}$ is the speed of light in free-space. Eliminating $E_{z}$ from this set, we obtain

$$
\left[\begin{array}{cc}
\Delta_{22}-\varepsilon_{z z} n^{2} & \Delta_{12} \\
-\Delta_{12} & \Delta_{11}-\varepsilon_{z z} n^{2}
\end{array}\right]\left[\begin{array}{l}
E_{x} \\
E_{y}
\end{array}\right]=0
$$

where $n=k_{z} c / \omega$ is the index of refraction and $\Delta_{i j}(i, j=1,2,3)$ is the co-factor of the element $\varepsilon_{i j}$ of the dielectric tensor $\hat{\varepsilon} ;$ e.g. $\Delta_{11}=\varepsilon_{y y} \varepsilon_{z z}-\varepsilon_{y z} \varepsilon_{z y}$, etc. In order to have a nontrivial solution for $\mathbf{E}$, the determinant of the left-hand matrix in Eq.(4) must vanish. The resulting equation, which determines $n$, is given by,

$$
\left(\varepsilon_{z z} n^{2}\right)^{2}-2 \Delta_{\times}\left(\varepsilon_{z z} n^{2}\right)+\left(\Delta_{11} \Delta_{22}+\Delta_{12}^{2}\right)=0
$$

where the abbreviation $\Delta_{x}=\left(\Delta_{11}+\Delta_{22}\right) / 2$ has been introduced. Now, the index of refraction can be found by solving Eq.(5) to give

$$
n_{1,2}^{2}=\left(\varepsilon_{z z}\right)^{-1}\left[\Delta_{\times} \pm\left(\Delta_{\times}^{2}-\varepsilon_{z z} \Delta\right)^{1 / 2}\right]
$$

where $\Delta=\operatorname{det}(\hat{\varepsilon})$ and the two solutions $n_{1}^{2}$ and $n_{2}^{2}$ correspond to indices of refraction of the two characteristic modes in the cold plasma. In the $x z$ plane the electric field vector of the two modes, $E_{\alpha}(\alpha=1,2)$ rotates on an ellipse. A relationship between $E_{x, \alpha}$ and $E_{y, \alpha}$ (wave polarization) is established in the waves of both modes from Eq.(4), that is

$$
\rho_{\alpha} \equiv\left(\frac{E_{x}}{E_{y}}\right)_{\alpha}=\frac{\Delta_{12}}{\Delta_{11}-\varepsilon_{z z} n_{\alpha}^{2}} .
$$

In view of the foregoing discussion, it is obvious that the most general plane wave solution has the following form,

$$
\mathbf{E}^{t}(\omega, z)=\hat{E}_{1} \exp \left(-j \omega k_{1} z\right)+\hat{E}_{2} \exp \left(-j \omega k_{2} z\right) \quad z>0
$$

where $\hat{E}_{1,2}$ are constant vectors, and $k_{1,2}=n_{1,2} \omega / c$. The magnetic field vector can be found from Maxwell's equations (1) to be

$$
\mathbf{H}^{t}(\omega, z)=\left(\omega \mu_{0}\right)^{-1}\left[k_{1} \hat{\mathbf{z}} \times \hat{E}_{1} \exp \left(-j \omega k_{1} z\right)+k_{2} \hat{\mathbf{z}} \times \hat{E}_{2} \exp \left(-j \omega k_{2} z\right)\right] \quad z>0
$$

From Eqs.(7) through (9), the explicit expressions for $E_{x}^{t}(\omega, z), E_{y}^{t}(\omega, z), H_{x}^{t}(\omega, z)$, and $H_{y}^{t}(\omega, z)$ may easily be obtained to be given by

$$
\begin{gathered}
E_{x}^{t}(\omega, z)=\hat{E}_{1} e^{-j \omega n_{1} z / c}+\hat{E}_{2} e^{-j \omega n_{2} z / c} \\
E_{y}^{t}(\omega, z)=\rho_{1} \hat{E}_{1} e^{-j \omega n_{1} z / c}+\rho_{2} \hat{E}_{2} e^{-j \omega n_{2} z / c}
\end{gathered}
$$




$$
\begin{gathered}
H_{x}^{t}(\omega, z)=-\frac{\rho_{1} n_{1}}{\eta_{0}} \hat{E}_{1} e^{-j \omega n_{1} z / c}-\frac{\rho_{2} n_{2}}{\eta_{0}} \hat{E}_{2} e^{-j \omega n_{2} z / c} \\
H_{y}^{t}(\omega, z)=\frac{n_{1}}{\eta_{0}} \hat{E}_{1} e^{-j \omega n_{1} z / c}+\frac{n_{2}}{\eta_{0}} \hat{E}_{2} e^{-j \omega n_{2} z / c}
\end{gathered}
$$

where $\eta_{0}=\left(\mu_{0} / \varepsilon_{0}\right)^{1 / 2}$ is the characteristic impedance of free-space and the unknown coefficinets $\hat{E}_{1,2}$ are to be found from the boundary conditions at the interface $z=0$.

\section{(b) Field components in the free space region}

Suppose that a linearly polarized wave with its electric field having only the $x$-component as its electric field is normally incident upon a plasma boundary. In the free-space region, the electric and magnetic fields are specified by Maxwell's equations (1) with $\hat{\varepsilon}$ replaced by a $3 \times 3$ unit matrix. The incident wave takes the following form

$$
\mathbf{E}^{i}(\omega, z)=\hat{\mathbf{x}} E_{0} e^{-j \omega z / c}, \quad \mathbf{H}^{i}(\omega, z)=\hat{\mathbf{y}} \frac{E_{0}}{\eta_{0}} e^{-j \omega z / c} \quad z<0
$$

where $E_{0}$ is the amplitude of the incident wave. The reflected wave is composed, in general, of two elliptically polarized components with different amplitudes, and is expressed in terms of a complex vector reflection coefficient $\mathbf{R}$ :

$$
\mathbf{E}^{r}(\omega, z)=\mathbf{R} e^{+j \omega z / c}, \quad \mathbf{H}^{r}(\omega, z)=\frac{\mathbf{R} \times \hat{\mathbf{z}}}{\eta_{0}} e^{+j \omega z / c} \quad z<0
$$

where the complex vector reflection coefficient $\mathbf{R}$ is necessary because of the transmittedwave characteristics and the necessity of matching the boundary conditions at $z=0$.

\section{(c) Boundary conditions}

The boundary conditions that the tangential components of the electric and magnetic field be continuous across the plane interface are imposed by matching the $x$ and $y$ components of $\mathbf{E}(\omega, z)$ and $\mathbf{H}(\omega, z)$ at $z=0$, that is

$$
\left.\begin{array}{rl}
E_{x}^{i}(\omega, 0)+E_{x}^{r}(\omega, 0) & =E_{x}^{t}(\omega, 0) \\
H_{y}^{i}(\omega, 0)+H_{y}^{r}(\omega, 0) & =H_{y}^{t}(\omega, 0) \\
E_{y}^{r}(\omega, 0) & =E_{y}^{t}(\omega, 0) \\
H_{x}^{r}(\omega, 0) & =H_{x}^{t}(\omega, 0)
\end{array}\right\} \quad z=0
$$

Substituiting Eqs.(10) through (15) in Eq. (16), we have

$$
\hat{E}_{1}=\frac{2 E_{0} N_{1}}{\left(1+n_{1}\right)}, \quad \hat{E}_{2}=-\frac{2 E_{0} N_{2}}{\left(1+n_{2}\right)}
$$

with

$$
N_{1}=\frac{\Delta_{11}-\varepsilon_{z z} n_{1}^{2}}{\varepsilon_{z z}\left(n_{2}^{2}-n_{1}^{2}\right)}, \quad N_{2}=\frac{\Delta_{11}-\varepsilon_{z z} n_{2}^{2}}{\varepsilon_{z z}\left(n_{2}^{2}-n_{1}^{2}\right)}
$$

and for the reflection coefficients $R_{x}=E_{1}+E_{2}-E_{0}, R_{y}=\rho_{1} E_{1}+\rho_{2} E_{2}$. The final task is to determine the time-dependent fields by taking the ILT of Eqs.(10) through (13), and 
(15) with the coefficients as given by Eqs.(16) and (17). The time-dependent transmitted and reflected electric field is then given in the following matrix form as,

$$
\|\tilde{\mathbf{E}}(t, z)\|=\left|\begin{array}{c}
\widetilde{E}_{x}^{t} \\
\widetilde{E}_{y}^{t} \\
\widetilde{E}_{x}^{r} \\
\widetilde{E}_{y}^{r}
\end{array}\right|=\frac{1}{2 \pi j} \int_{B r}\|\mathbf{E}(s, z)\| e^{s t} d s
$$

where $s=j \omega$ is the Laplace transform variable and $B r$ defines a Bromwich path in the complex $s$-plane. These integrals cannot be evaluated in a closed form, but a direct numerical integration is possible since the integrands are real functions of $s$ for real $s$.

\section{Numerical Results and Discussion}

The transient field components $\widetilde{\mathbf{E}}(t, z)$ were evaluated using the formula of numerical inversion of the Laplace transform as proposed by Hosono (1981). The ionosphere is modeled as a two-component collisional (cold) plasma consisting of electrons and oxygen ions, $O^{+}$. Table 1 summarizes the plasma parameters representing the daytime $F_{2}$ layer ionosphere at a height of about $350 \mathrm{~km}$ which are adopted from Al'pert (1990). In this table $f_{p e}$ and $f_{c e}$, the electron plasma and electron cyclotron frequencies; $f_{p i}$ and $f_{c i}$, the ion plasma and ion cyclotron frequencies; $f_{R}$ and $f_{L}$, the cutoff frequencies for rightand left- hand polarized waves; $f_{U H R}$ and $f_{L H R}$, the upper- and lower-hybrid resonance frequencies; and $\nu_{e}$ and $\nu_{i}$, the collision frequencies of the electrons and ions with the neutral particles (molecules). Based on the parameters given in Table 1, we discuss the numerical results in three different frequency bands as follows: (i) quasi-free space mode waves $|\operatorname{Im}(s)|>\omega_{L} / \omega_{p e} \simeq 1.0$; (ii) whistler mode wave $|\operatorname{Im}(s)|<\omega_{c e} / \omega_{p e} \simeq 0.1$; and (iii) hydromagnetic waves $|\operatorname{Im}(s)|<\omega_{c i} / \omega_{p e} \simeq 3.4 \times 10^{-6}$. Particular attention will be given to the effect of oblique orientation of Earth's magnetic field $\mathbf{B}_{0}$ on the transient signals.

In the subsequent calculations, we introduce the dimensionless quantities $\mathrm{S}=s / \omega_{p e}, \tau=$ $\omega_{p e} t$, and $\xi_{z}=z \omega_{p e} / c$. All the parameters listed in Table 1 are normalized accordingly; e.g. $\Omega=\omega / \omega_{p e}, \Omega_{c e}=\omega_{c e} / \omega_{p e}, \Omega_{p i}=\omega_{p i} / \omega_{p e}, \bar{\nu}_{e}=\nu_{e} / \omega_{p e}$, etc. Consequently, the "vertical " axis represents a dimentionless electric field defined as $E_{x, y}\left(\tau, \xi_{z}\right) / \omega_{p e} E_{0}$, where $\mathrm{E}_{0}$ is the amplitude of the incident wave in Eq.(6). The "horizantal" axis corresponds to the dimentionless time $\tau\left(=\omega_{p e} t\right)$. The value of $\xi_{z}\left(=z \omega_{p e} / c\right)$ in each figure is constant. For $f_{p e}=10 \mathrm{MHz}, \tau=1$ implies that $t=1 \mu \mathrm{s}$, while $\xi_{z}=200$ implies $z=955 \mathrm{~m}$, etc.

Since the dispersion results from the transmission effect at the interface $z=0$ as well as propagation effect within the medium $z>0$, we shall examine both cases in the followings.

\subsection{Computational results of free-space wave modes $\left(|\operatorname{lm}(S)|>\Omega_{L} \simeq 1.0\right)$}

This range corresponds to the high frequency, quasi-free space mode waves for which the ions play a very little role due to their large mass. The numerical results in this frequency range are shown in Figs. 2 and 3. In these figures, the top panel refers to the co-polarized component, $\widetilde{E}_{x}\left(\tau, \xi_{z}\right)$, and the bottom panel gives the corresponding crosspolarized component, $\widetilde{E}_{y}\left(\tau, \xi_{z}\right)$. Fig.2 illustrates the time-dependent reflected/transmitted waveforms at the interface. The waveforms for $\xi_{z}=0$ yield the dispersion of the normally incident impulsive plane wave due to the transmission effect only which occurs at the interface. One should notice that in the absense of the magnetic field $\mathbf{B}_{0}$, the cross- 


\section{Quasi-free space modes, $\xi_{2}=0$}

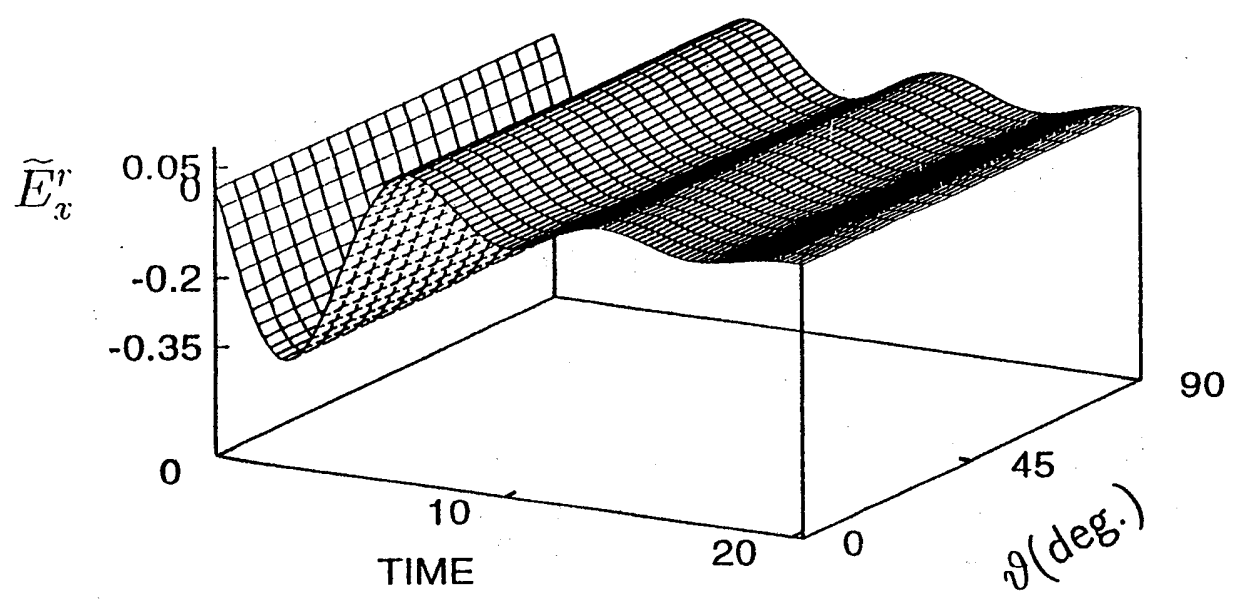

(a)

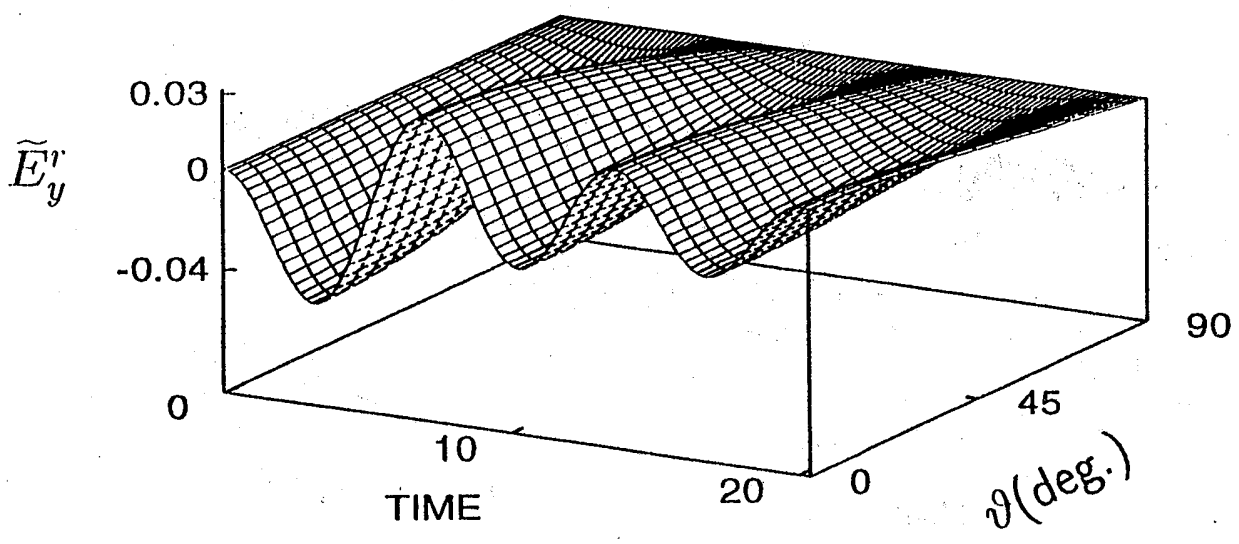

(b)

Fig. 2 Time-dependent co- and cross-polarized components $\widetilde{E}_{x, y}^{r}\left(\tau, \xi_{z}\right)$ of the reflected quasi-free space mode waves with $\xi_{z}=0$ as a function of $\vartheta$. 
Quasi-free space modes, $\xi_{z}=7$

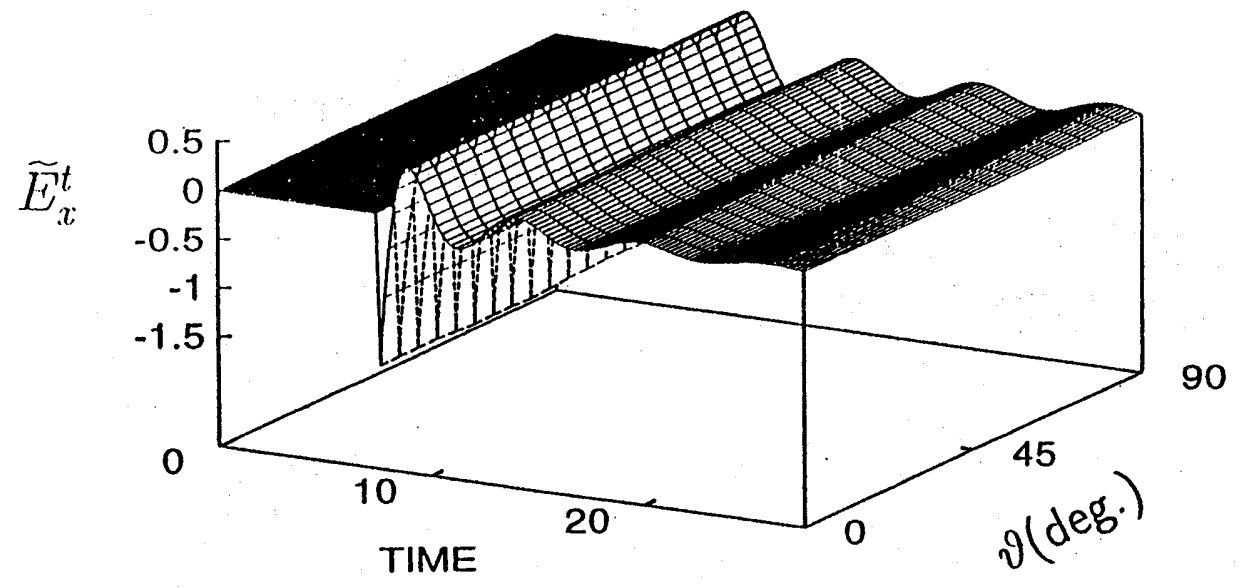

(a)

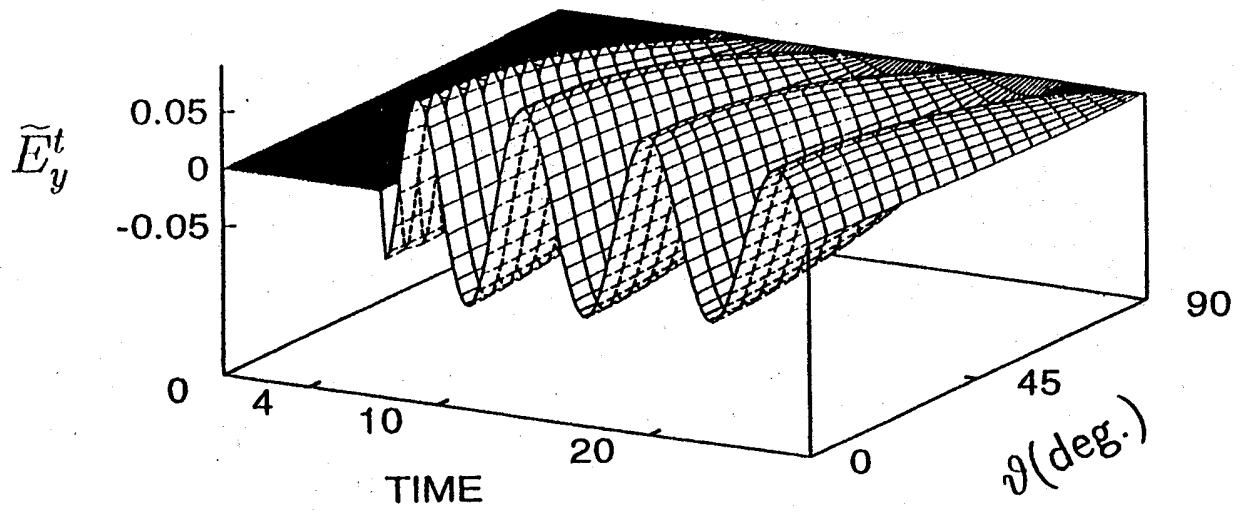

(b)

Fig. 3 Time-dependent co- and cross-polarized components $\tilde{E}_{x, y}^{t}\left(\tau, \xi_{z}\right)$ of the transmitted quasi-free space mode waves at $\xi_{z}=7$ as a function of $\vartheta$. 
polarized components $\widetilde{E}_{y}^{t}$ and $\widetilde{E}_{y}^{r}$ would be zero. On the other hand, as $\omega_{c e}$ takes on a greater value, a greater part of energy goes to the cross-polarized component. In order to examine the propagation effect, the time-dependent field components at one particular distance $\left(\xi_{z}=7\right)$ from the interface are illustrated in Fig.3. It can be seen that the waveforms are growing as $\xi_{z}$ increases which correspond to an energy transfer from the $\widetilde{E}_{x}^{t}$ component to the $\tilde{E}_{y}^{t}$ component. The major change which occurs when increasing $\nu_{e}$ is to reduce the amplitude of the field components, with the frequency of oscillatory response being practically uneffected as shown in Stanic and Skoric (1973).

\subsection{Whistler branch $\left(|\operatorname{Im}(S)|<\Omega_{c e} \simeq 0.1\right)$}

In this frequency band only one wave is propagating, namely, whistler-mode. The computational results of the waveforms in this frequency range are shown in Fig. 4 . We have computed the waveforms of whistler-mode waves at one particular distance $\left(\xi_{z}=400\right)$ from the interface, and three different $\vartheta$ values are adopted; $\vartheta=0^{\circ}$ (a), $25^{\circ}$ (b) and $50^{\circ}$ (c). In this case, $\xi_{z}>0$, the transmitted waveforms are further dispersed due to the propagation effect. Since $\xi_{z}=400>>0$, the plasma medium can be considered as infinite. In this way, the propagation effect by itself can be examined. The most conspicuous point of Fig. 4 is the rapid increase of the amplitude, which is closely related to the wave components with frequencies near a quarter of electron gyro-frequency $\Omega_{\text {nose }}=\Omega_{c e} \cos \vartheta / 4$ because the group velocity at $\Omega_{\text {nose }}$ attains its maximum value in the case of a homogeneous magnetoplasma (Omid and Hayakawa, 1994). This frequency is sometimes called a "nose frequency" in the whistler spectrum (Helliwell, 1965; Hayakawa, 1995). The subsequent modulation envelopes as seen in these figures are resulted from the interference between the wave components above and below this frequency,$\Omega_{\text {nose }}$, whose group velocities are close to each other. Although the wave components with $\Omega>\Omega_{\text {nose }}$ are shown later to decay more quickly due to the collisional damping than those at $\Omega<\Omega_{\text {nose }}$ (Helliwell,1965; Hayakawa, 1995), the response becomes less oscillatory and smooths out the modulation envelope, at shorter distances (smaller $\xi_{z}$ ) from the interface (see Fig.5 of Omid and Hayakawa, 1994) because the high-frequency wave components contain more energy than the low-frequency ones and hence dominate the response (Vidmar et al., 1983). Further effects which an oblique Earth's magnetic field has on the waveforms as inferred from Fig. 4 are as follows: (a) Longer delay in the arrival of wave packets

For constant $\xi_{z}$ the maximum group delay, $\tau_{d m}$, is proportional to $[\cos \vartheta]^{-1}$ (see Omid and Hayakawa, 1994, Eq.28) and it would be approximately equal to 6160,6800 and 9580 , respectively, at $\xi_{z}=400$ for $\vartheta=0^{\circ}, 25^{\circ}$ and $50^{\circ}$. This expectation is consistent with the larger time delay in the arrival of wave packets with respect to $\vartheta$ as indicated by the triangles in Fig.4. Notice that the ratio of $\xi_{z}$ to $\tau$ in each panel corresponds to the group velocity normalized to the speed of light, i.e. $\xi_{z} / \tau=V_{g z} / c$. The curves to the left of the triangles in Fig.4 are whistler branch precursors, which propagate faster than $V_{g m}$ (Vidmar, et al., 1983).

(b) More reduced amplitude for larger $\vartheta$ 's

The decrease of amplitude with respect to $\vartheta$ as found in Fig.4 can be explained in terms of the enhanced attenuation for larger $\vartheta$ values. It has been shown that the reduced intensity is related to $\vartheta$ with the relationship of $1 /(\cos \vartheta)^{1.5}$ (Omid and Hayakawa, 1994). 


$$
\text { Whistler, } \xi_{z}=400
$$
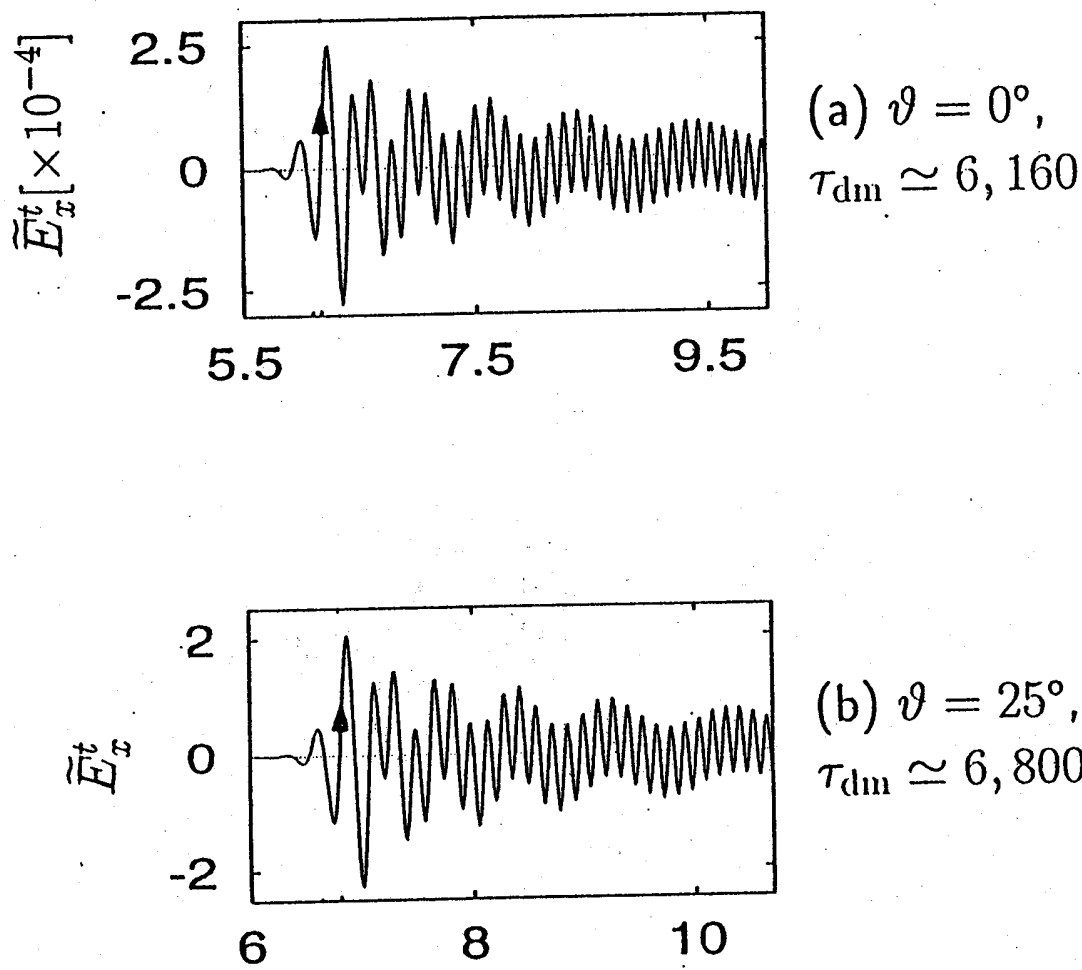

(b) $\vartheta=25^{\circ}$,

$\tau_{\mathrm{dm}} \simeq 6,800$

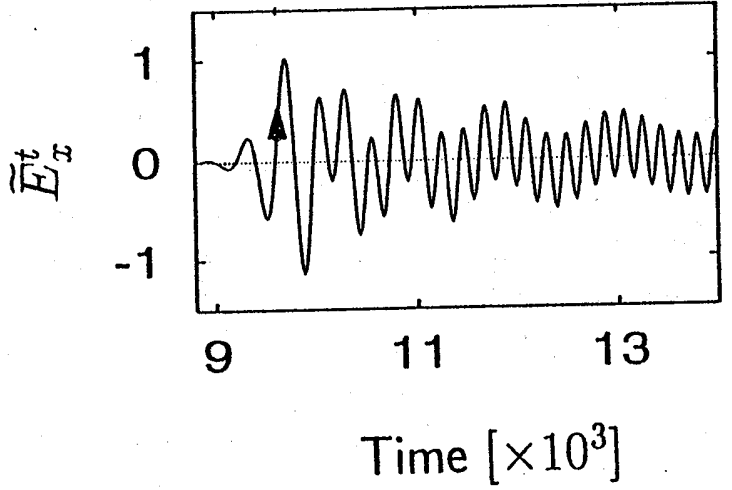

(c) $\vartheta=50^{\circ}$,

$\tau_{\mathrm{dm}} \simeq 9,580$

Fig. 4 Time-dependent co-polarized component $\tilde{E}_{x}^{t}\left(\tau, \xi_{z}\right)$ of the transmitted whistler wave at $\xi_{z}=400$ as a function of $\vartheta$. (a) $\vartheta=0^{\circ}$, (b) $\vartheta=25^{\circ}$ and (c) $\vartheta=50^{\circ}$ 


\subsection{Computational results of hydromagnetic wave modes}

$\left(|\operatorname{lm}(S)|<\Omega_{c i} \simeq 3.4 \times 10^{-6}\right)$

The computational results of the waveforms in this frequency range (hydromagnetic waves) are shown in Fig.5. The co-polarized component $\widetilde{E}_{x}^{t}\left(\tau, \xi_{z}\right)$ and cross-polarized one $\widetilde{E}_{y}^{t}\left(\tau, \xi_{z}\right)$ at $\xi=20,000$ for $\theta=0^{\circ}$ are superimposed in Fig.5(a). The two propagating modes with opposite polarization are propagated, i.e. modified-Alfven wave (whistlermode) and Alfven wave (ion cyclotron wave). As $\omega$ goes to zero $\left(t \rightarrow t_{\Delta}\right)$, the modifiedAlfven wave propagates nearly at the Alfven velocity $v_{A}$ and the Alfven wave propagates with group velocity slightly less than $v_{A}$ (Vidmar, et al., 1983). The phase difference in Fig.5(a) shows that prior to the arrival of zero frequency components (i.e. $\tau<\tau_{\Delta}$ ) we have a right-handed circular polarization (modified Alfven wave), while a left-handed circular polarization (Alfven wave) is propagating afterward(i.e. $\tau>\tau_{\triangle}$ ). After the arrival of zero frequency components, the amplitude of the waveform decreases while its frequency increases to $\Omega_{c i}$. The characteristic dip (CD) at $\tau=\tau_{\triangle}$ is the consequence of interference of the two waves with opposite polarization, and for $\vartheta=0^{\circ}$ this CD occurs at $\tau=\tau_{\triangle}$ and can be used to estimate the Alfven velocity and hence plasma parameters (Vidmar, et al., 1983). In Figs.5(b) and (c) we illustrate the waveforms for co-polarized component of $\widetilde{E}_{x}\left(\tau, \xi_{z}\right)$ at $\xi_{z}=20,000$ with $\vartheta=25^{\circ}$ and $50^{\circ}$, respectively. The major changes accomplished by increasing $\vartheta$ can be summarized as: (a) longer group delay in Alfven wave and (b) gradual decrease in Alfven wave amplitude. It can be shown (see, for example, Booker, 1984) that the refractive index and hence the group velocity of the modified-Alfven wave is independent of $\vartheta$ angle, and so the increase in $\vartheta$ would result in increase of frequency of oscillation only and would not have any effect on the amplitude of waveforms for constant $\xi_{z}$. At smaller $\xi_{z}$ the modified-Alfven wave dominates the response. On the contrary, the behavior of Alfven wave packets is very similar to that of the whistlermode wave. With the increase of $\vartheta$, the initial part of the Alfven wave is more delayed, and its amplitude is reduced due to absorption just like the case of whistler propagation.

\section{Conclusions}

We have studied numerically the transient reflection and transmission of an impulsive plane wave without making any approximation for a half-space magnetoplasma when the earth's magnetic field is allowed to be oriented arbitrarily. The results serve as a special illustration of cold plasma theory and can be used as a plasma diagnostic tool by relating cold plasma theory to specific features in a waveform, e.g. plasma parameters such as the plasma frequency, electron and ion cyclotron frequencies, distance from the interface and wave polarization can be deduced. We can list some main effects of Earth's magnetic field orientations as follows: For the whistler wave, arrival of signal delay is inversley proportional to $\cos \vartheta$ and and its reduced intensity is expected for larger $\vartheta$ 's. And, for the hydromagnetic wave modes, the two-oppositely polarized waves are excited( namely, modified-Alfven wave and Alfven wave), with the latter being very similar to the whistler case, whereas the former one, not much effected although the frequency of its oscillation increases as the $\vartheta$ increases.

Acknowledgements. One of the authors (M.O.) greatfully acknowledges the support of the Iranian Ministry of Culture and Higher Education and a research grant from the Japanese Government. 
Hydromagnetic waves,
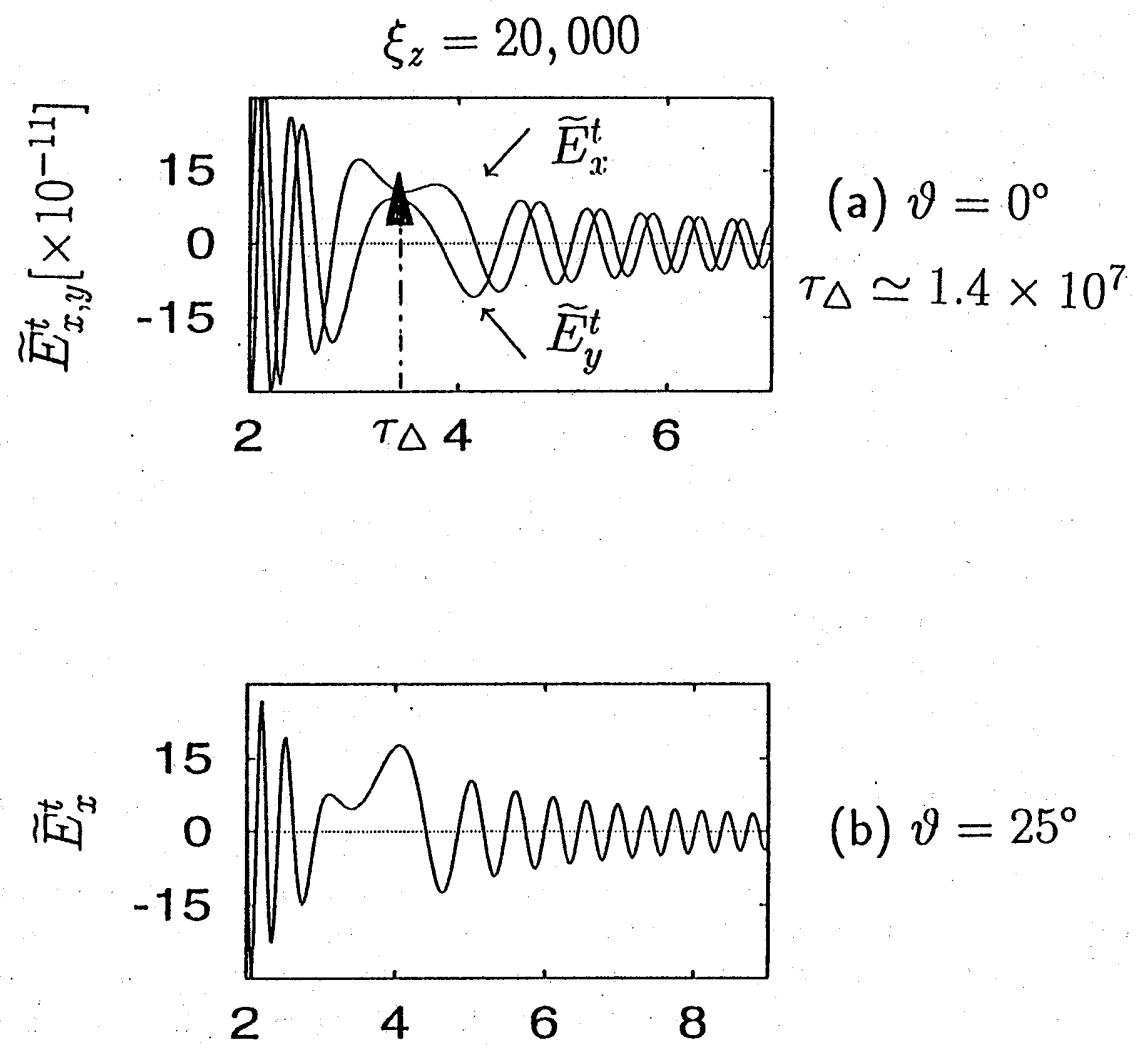

(b) $\vartheta=25^{\circ}$

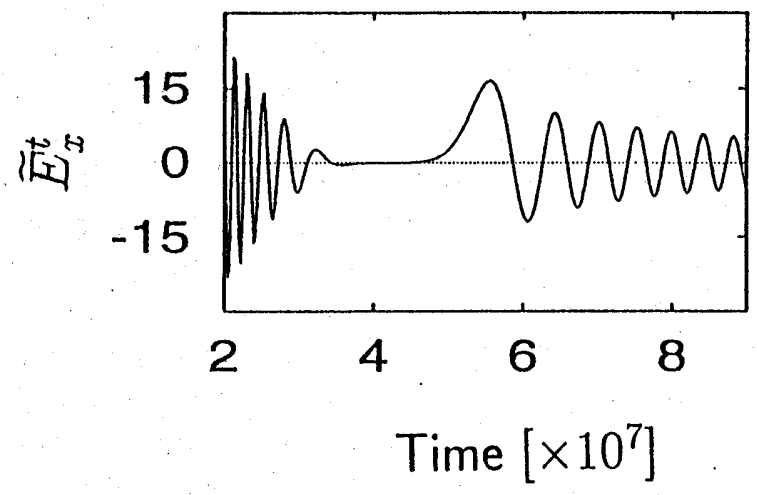

(c) $\vartheta=50^{\circ}$

Fig. 5 (a) Time-dependent co- and cross-polarized components $\widetilde{E}_{x, y}^{t}\left(\tau, \xi_{z}\right)$ of the transmitted hydromagnetic waves at $\xi_{z}=20,000$ and $\vartheta=0^{\circ}$; (b) co-polarized component of $\widetilde{E}_{x}^{t}\left(\tau, \xi_{z}\right)$ with $\vartheta=25^{\circ}$ and (c) co-polarized component of $\widetilde{E}_{x}^{t}\left(\tau, \xi_{z}\right)$ with $\vartheta=50^{\circ}$ 


\section{References}

Al'pert Y.L. , Space Plasma; Vol.I: Theory and Main Properties, Cambridge Univ. Press, Cambridge, 1990.

Booker H.G. , Cold Plasma Waves, Martinus Nijhoff Pub., Dordrecht, 1984.

Budden K.G. , The Propagation of Radio Waves, Cambridge Univ. Press, Cambridge, 1985.

Hayakawa M. , Whistlers, In Handbook of Atmospheric Electrodynamics, CRC Press, Florida, Vol.II, 155-193, 1995.

Helliwell R.A. , Whistlers and Related Ionospheric Phenomena, Stanford Univ. Press, Stanford, 1965.

Hosono T. , Numerical inversion of Laplace transform and some applications to wave optics, Radio Sci.,16, 1015-1019, 1989.

Hosono T., T. Hinata, and H. Kishi , Transient responses of electromagnetic waves in magnetized plasma by plane wave excitation, Proc. Int'l Symp. Ant. Prop. (ISAP'89), Tokyo, 743-746, 1989.

Ishimaru A. , Transients in magneto-ionic media and interactions between ordinary and extraordinary wave representations, in Electromagnetic Wave Theory, $\underline{2}$, Ed. by J. Brown, Pergamon Press, New York, 125-134, 1967.

Omid M. and M. Hayakawa , Propagation of transient signals in a magnetoactive plasma, Proc. Int'l Symp. Ant. Prop. (ISAP'92), Sapporo, 589-592, 1992.

Omid M. and M. Hayakawa, Excitation of electromagnetic waves by delta function current sheets in the ionospheric plasma, Radio Sci., 29 $, 867-877,1994$.

Omid M., T. Yoshino, and M. Hayakawa , Transient propagation of EM waves in a magnetoactive plasma, Proc. Int'l Symp. Radio Prop. (ISRP'93), Beijing, 240-243, 1993.

Stanic B.V. and M.M. Skoric, Collisional damping of an impulsive plane wave incident on an anisotropic plasma half-space, J.Phys.D, $\underline{6}, 165-167,1973$.

Vidmar R.J., F. W. Crawford, and K. J. Harker, Delta function excitation of waves in the earth's ionosphere, Radio Sci., 18, 1337-1354, 1983.

(Received May 14, 1996; revised July 9, 1996;

accepted July 12, 1996.) 